\title{
Using routinely collected data to improve immunisation histories
}

\author{
Luke Allen \\ Royal Cornwall Hospital
}

\begin{abstract}
Immunisation is one of the most effective health interventions in existence yet outbreaks of vaccine-preventable diseases continue to occur in developed countries. High rates of cover are needed to provide adequate herd immunity and there is evidence that a significant proportion of paediatric inpatients are not up to date even in areas with high levels of community cover. A proportion of these children will have parents who consciously declined immunisation, however the remaining children represent a vulnerable cohort whose under-immunisation is not routinely identified.
\end{abstract}

Two-hundred consecutive admissions to rural paediatric assessment unit had their routinely documented vaccination histories checked against their records on the regional Child Health Information System (CHIS). 30 children (15\%) were not up to date on the CHIS, yet routine clerking only identified 5 of these children (17\%). After introduction of a simple system whereby ward clerks and doctors were taught how to access and print full immunisation histories from the CHIS, a further 200 consecutive admissions were audited. A similar number were not up to date (29/200) but the proportion of children with missing immunisations correctly identified in the clerking documentation increased to $52 \%$ (15 children). This is a $35 \%$ improvement $(95 \% \mathrm{Cl} ; 12-58 \%)$.

Access to routinely collected data can significantly improve identification of under-immunised children and contribute towards higher levels of individual and herd-immunity.

\section{Problem}

Recent outbreaks of vaccine-preventable diseases in England and Wales highlight the importance of ensuring that paediatric inpatients are up to date with their immunisations. Standard vaccination history taking at the Royal Cornwall Hospital generally constitutes a cursory verbal enquiry invariably resulting in a three letter entry in the notes - "utd". Along with Walton et al. [1] I felt this to be a generic problem.

Up to $40 \%$ of patients presenting to urban centres lack one or more immunisations [1-5] and I was concerned that we may be missing patients who were vulnerable to vaccine-preventable diseases. Verbal immunisation history-taking is very much the status-quo, despite evidence that the use of computer records can help. [1,6-7] Ubiquitous constraints on time, the ever-evolving immunisation schedule, and imperfect parental-recall also hinder the identification of missed doses and full documentation of immunisation histories.

\section{Background}

Immunisation is one of the most effective health interventions in existence yet outbreaks of vaccine-preventable diseases continue to occur even in developed countries. [8] High rates of cover are needed to provide adequate herd immunity and there is evidence that a significant proportion of paediatric inpatients are not up to date even in areas with relatively high levels of community cover. $[1,4]$

In every country a proportion of parents may decline immunisation for their children, however, studies consistently demonstrate the existence of a cohort of children who are amenable to immunisation when offered in the clinical setting, but for one reason or another do not actively seek immunisation and are not identified by routine immunisation history-taking. [1-5, 9]

The use of regional Child Health Information Systems (CHISs) which hold individual immunisation records has been advocated as a means of improving the quality of immunisation histories for decades. $[1,5]$ Several studies in urban paediatric centres have successfully used CHIS-based systems to improve identification of under-immunised patients, $[1,3,-4]$ however there is no published evidence from more rural areas with higher rates of cover.

\section{Baseline Measurement}

To establish the extent of the problem, the CHIS records of 200 consecutive admissions to the paediatric assessment unit were compared with these patient's documented immunisation histories. Thirty patients (15\%) were documented as missing one or more immunisation on their $\mathrm{CHIS}$ record, however only $5(17 \%)$ of these were correctly identified in the clerking notes. $4(13 \%)$ of the underimmunised patents had the wrong immunisations documented as missing. None of these patients had a full immunisation history recorded in the clerking documentation and $8(38 \%)$ had no immunisation information documented at all. 9 (33\%) were documented as being "up to date".

\section{Design}


After discussion with the child health records department I obtained access to the regional CHIS, which contains full immunisation histories for every child in the county. I trained ward-clerks to use the system, and with consultant and ward-manager approval they were asked to print each admission's full vaccination history and file it with the clerking documents for reference. Paediatricians were advised to use the printouts when clerking new patients and I also trained them to access the CHIS when ward-clerks were not available. The current UK immunisation schedule was made available in paper and electronic formats. I discussed the system before implementation at a team business meeting to address any queries.

Similar systems have been used to good effect in the studies referenced above and tallied with colleagues' experiences in New Zealand and centres in other countries around the globe. As it takes approximately 2 weeks for an immunisation to be registered on the system and occasional errors still occur, the CHIS printout should augment rather than replace parental history. Accepting these two limitations, printed CHIS records are otherwise quickly obtained and provide a complete and accurate immunisation record.

\section{Strategy}

The intervention was first mooted at a journal-club meeting with senior paediatric staff in the context of a review of a similar project at Great Ormond Street Hospital. Initial feedback highlighted several barriers experienced by other authors. Some staff felt immunisation to lie beyond the remit of secondary care, others felt $\mathrm{CHIS}$ records would be inaccessible, and concerns were raised that the system would deliver little benefit due to high levels of community cover.

Results from the baseline audit galvanised support for the intervention which was refined through discussion with consultants, the ward manager, and ward-clerks. Responsibility for accessing and printing the CHIS immunisation histories was given to the ward clerks who were familiar with the software and already had responsibility for assembling the clerking documentation. It was agreed to trial the system for 3 weeks and then re-audit to examine efficacy before permanently adopting the new system.

The system was presented to junior staff at a teaching session a week before implementation and again to the whole team at a business meeting on the morning of the first day. Following feedback from these meetings the current UK immunisation schedule was made available on posters in the doctors' office and electronically on a shared drive.

\section{Results}

One week after the new system was introduced the $\mathrm{CHIS}$ records of a further 200 consecutive admissions to the paediatric assessment unit were compared with the clerking documentation. A comparable number of admissions were documented as lacking one or more immunisations on the CHIS (29/200, 15\%). 15 of these 29 had their missing immunisations correctly documented in the notes. This represents a $35 \%$ increase in positive identification of underimmunised patients (95\% Cl; 12-58\%). Eight (28\%) of the 29 patients had a copy of the full vaccination history in the clerking documents compared with 0 at baseline. This represents a $28 \%$ increase (95\% Cl;12-44\%).

\section{Lessons and Limitations}

Exploring and engaging with staff concerns was an important element of this project, as was identifying key members the medical, nursing, pharmaceutical, and administrative teams. Having local evidence from the baseline audit to augment national evidence was invaluable in motivating these individuals to drive the changes amongst their peers. The project served to highlight the vast amounts of data that is routinely collected but seldom used and emphasised how usefully this information can be employed.

The project was limited by the short time interval between implementation and re-audit which may over-estimate the effect in the medium to long term. Doctors seldom printed CHIS immunisation histories themselves and subsequently 'out of hours' immunisation histories were of a poorer quality. Extending wardclerk hours has been suggested by the ward manager which would have a positive impact but the bedding in of cultural change would be far more constructive.

\section{Conclusion}

The pervasive, if not ubiquitous problem of incomplete and inaccurate immunisation histories, systematically discriminates against a small but significant group of under-immunised patents. Utilising readily-available CHIS data can significantly improve the quality of immunisation histories in clinical settings, contributing towards a reduction in vaccine-preventable disease susceptibility for individuals and increased herd-immunity for populations.

\section{References}

1. Conway S. Opportunistic immunisation in hospital. Arch Dis Child. 1999;(81):422-5.

2. Gandhi M, McKenna S, Geraets A, Bhatt R, Greenberg M, Bartley A, et al. Establishing an opportunistic catch up immunisation service for children attending an acute trust in London. Arch Dis Child. 2011;(96):780-781.

3. McKinlay A, Rankin W. Hospital admission - a missed opportunity to immunise [letter]. Arch Dis Child. 1991;(66(2)):273.

4. Riley D, Mughal M, Roland J. Immunisation state of young children admitted to hospital and effectiveness of a ward based opportunistic immunisation policy. BMJ. $1991 ;(302): 31-3$.

5. Walton S, Elliman D, Bedford H. Missed opportunities to vaccinate children admitted to a paediatric tertiary hospital. Arch Dis Child. 2007;(92):620-2.

6. Amirthalingam G, White J, Ramsay M. Measuring childhood vaccine coverage in England: the role of Child Health Information Systems . Euro Surveill. 2012;(17(16)):20149. 


\section{BMJ Quality Improvement Reports}

7. Myrick C. Shinall, Jr, Erin J. Plosa and Katherine A. Poehling. Validity of Parental Report of Influenza Vaccination in Children 6 to 59 Months of Age. Pediatrics. 2007;(120);783

8. Council for Foreign Relations (CFR). Vaccine-Preventable Outbreaks Map. [Available at] http://www.cfr.org/interactives/GH Vaccine Map/index.html \#map [Accessed 18/9/2013].

9. Berling I, Stephenson J, Cashman P, Loten C, Butler M, Durrheim D. Opportunistic childhood vaccinations in emergency -Are we really missing anyone? Australasian Emergency Nursing Journal. 2012;(15):37-44.

\section{Declaration of interests}

Nothing to declare.

\section{Acknowledgements}

Helen Vickerstaff, Carol Matthews 